Fi ni te El ement Nodel for Spot Wel ds Usi ng Mil ti - Poi nt Const rai nt s and i ts Dynami $\mathrm{c}$ Char act er i st i cs

\begin{tabular}{|l|l|}
\hline $\begin{array}{l}\text { jour nal or } \\
\text { publ i cat i on titl e }\end{array}$ & $\begin{array}{l}\text { Proceedi ngs of SAE 2011 Noi se and Vi br at i on } \\
\text { Conf er ence and Exhi bi t i on }\end{array}$ \\
\hline vol une & 2011 \\
\hline page range & $01-1697$ \\
\hline year & $2011-05$ \\
\hline URL & ht t p: //hdl . handl e. net /10098/5248 \\
\hline
\end{tabular}




\title{
Finite Element Model for Spot Welds Using Multi- Point Constraints and its Dynamic Characteristics
}

\author{
Fumiyasu Kuratani, Kazuhei Matsubara and Takashi Yamauchi \\ University of Fukui
}

\begin{abstract}
In this paper, we focus on the ACM2 (Area Contact Model 2) model as a simplified spot weld model suitable for vibration analysis and reveal its dynamic characteristics. First, the theoretical background of the multi-point constraint (MPC) used in the ACM2 model is explained. Next, we examine in detail the effect of the mesh pattern surrounding a spot weld on the modal properties (natural frequency and mode shape) of a spot welded structure. Finally, an appropriate mesh size in the area of the spot weld for the ACM2 model is presented. As an example, we used two steel plates joined by three spot welds.
\end{abstract}

The results show that the configuration and size of the patch (group of shell elements in the ACM2 model) significantly affect the modal properties of the model. When the centers of the patch and the solid element that represent the spot weld are coincident, the natural frequencies monotonically increase with the patch size. When the centers of the patch and the solid element are not coincident and the shell element size is large, the natural frequencies vary widely. When the patch is composed of shell elements whose size is the same or smaller than the solid element size determined by the spot weld diameter, the variation in the natural frequency is small.

\section{INTRODUCTION}

Spot welds are widely used in the automotive industry to join thin sheet metal. Since a typical automotive body contains several thousand spot welds, its structural behavior is strongly affected by the characteristics of spot welded joints. Appropriate modeling of spot welded joints is essential when analyzing structural behavior using finite element analysis (FEA). For the stress analysis of spot welded joints, a very detailed model is necessary and various finite element (FE) models have been proposed [1, 2$]$. For the vibration analysis of a large scale model such as an automotive body, the use of a detailed model is impractical because a model with large degrees of freedom (DOF) leads to excessive computational effort. Thus the major requirements of spot weld models for vibration analysis are to represent the stiffness of spot welds with small DOF and to predict accurately their influence on the rest of the structure. In addition, it is important that spot weld models are easy to generate and the locations of spot welds are easily modified. That is, the joined metal plates are meshed independently of the spot weld locations and the spot welds can be located anywhere in the mesh of the plates.

For the above requirements, Heiserer et al. [3] proposed the model known as ACM2 (Area Contact Model 2) and Jonscher et al. [4] proposed the CWELD model. The ACM2 model was developed using the finite element program NASTRAN and is the most commonly used by Japanese automotive manufacturers. For the ACM2 model, a spot weld is modeled with a single solid element and the plates surrounding the spot weld are modeled with shell elements. The solid element node and the shell element nodes are connected using multi-point constraints (MPC). This enables the spot welds to be located anywhere in the mesh of the plates. Some spot weld models, including the ACM2 model, were examined for their effect on the vibration properties of a spot welded structure $[\underline{5}, \underline{6}, \underline{7}]$. However, the dynamic characteristics of the ACM2 model remain to be fully elucidated.

In this paper, we focus on the ACM2 model as a simplified spot weld model for vibration analysis and reveal its dynamic characteristics. First, the theoretical background of the MPC used in the ACM2 model is explained. Next, we examine in detail the effect of the mesh pattern surrounding a spot weld on the modal properties (natural frequency and mode shape) 
of a spot welded structure. This determines the factors of the MPC equations. Finally, an appropriate mesh size in the area of the spot weld is presented. In this paper, using the finite element program ANSYS we create a finite element model for spot welds with the same features as the ACM2 model. As an example, we used two steel plates joined by three spot welds.

\section{SPOT WELD MODEL}

In the vibration analysis of a large scale spot welded structure, a simplified model for spot welds is needed. However, a model using a single beam element or a single solid element to represent a spot weld requires the matching of meshes of different plates at the spot weld connection points. This results in excessive modeling effort. In addition, a single beam model underestimates the stiffness of a real spot welded joint due to the point-to-point joint [6]. Thus the major requirements of spot weld models are to represent the stiffness of spot welds with small DOF and to predict their influence on the rest of the structure accurately. In addition, the joined metal plates are meshed independently of the spot weld locations and the spot welds can be located anywhere in the mesh of the plates.

The CWELD and the ACM2 models meet the above requirements. The CWELD model is provided as the CWELD element in NASTRAN while the ACM2 model is generated using several types of elements in NASTRAN. The ACM2 model is the most commonly used by Japanese automotive manufacturers.

\section{ACM2 MODEL}

In this paper, we focus on the ACM2 model as a spot weld model as shown in Figure 1. For the ACM2 model, a spot weld is modeled with a single solid element and the upper and lower plates are modeled with shell elements. The solid element node and the shell element nodes are connected using RBE3 (Rigid Beam Element 3) elements. This enables spot welds to be located anywhere independently of the mesh of the plates. The RBE3 element is available in NASTRAN and generates MPC equations that define the relationship between the solid element node and the shell element nodes. The factors of MPC equations are determined on the basis that the translational force applied to the solid element node is distributed to the shell element nodes. The group of shell elements in the ACM2 model is called a patch.

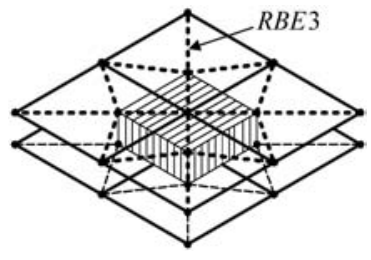

Figure 1. ACM2 model.

\section{MPC EQUATION USING THE RBE3 ELEMENT}

The MPC equation defines a linear relationship between the displacement of nodes within a FE model. Figure 2 shows the connections between one solid element node and four shell element nodes with four RBE3 elements. Since the five nodes in the ACM2 model lie in the same plane, the translations in the $x, y$ and $z$ directions at the solid element node are represented by the translations in the $x, y$ and $z$ directions at the four shell element nodes using MPC equations, that is

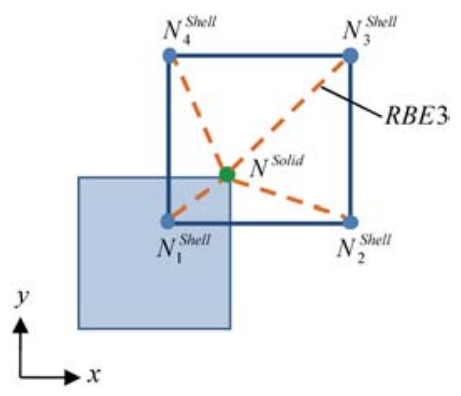

Figure 2. Solid element node and shell element nodes connected with RBE3 elements.

$$
\begin{aligned}
& u_{x}^{\text {Solid }}=\sum_{i=1}^{4} \alpha_{x i} u_{x i}^{\text {Shell }}+\sum_{i=1}^{4} \alpha_{y i} u_{y i}^{\text {Shell }} \\
& u_{y}^{\text {Solid }}=\sum_{i=1}^{4} \beta_{x i} u_{x i}^{\text {Shell }}+\sum_{i=1}^{4} \beta_{y i} u_{y i}^{\text {Shell }} \\
& u_{z}^{\text {Solid }}=\sum_{i=1}^{4} \gamma_{z i} u_{z i}^{\text {Shell }}
\end{aligned}
$$

where $u_{x}^{\text {Solid }}, u_{y}^{\text {Solid }}, u_{z}^{\text {Solid }}$ are the translations of the solid element node. $u_{x}^{\text {Shell }}, u_{y}^{\text {Shell }}, u_{z}^{\text {Shell }}$ are the translations of $i$ th shell element node. $\alpha_{x i}, \alpha_{y i}, \beta_{x i}, \beta_{y i}, \gamma_{z i}$ are the factors of the MPC equations. The mathematical specification for the MPC equation generated by the RBE3 element is undocumented. We present a method for determining the factors of the MPC equations based on Nastran Reference Manual []].

The MPC equations in Equation (1) are defined in the three translational directions. The case of $\alpha_{x i}$ and $\alpha_{y i}$ is demonstrated here. An in-plane force in the $x$ direction is applied to the solid element node as shown in Figure 3. 


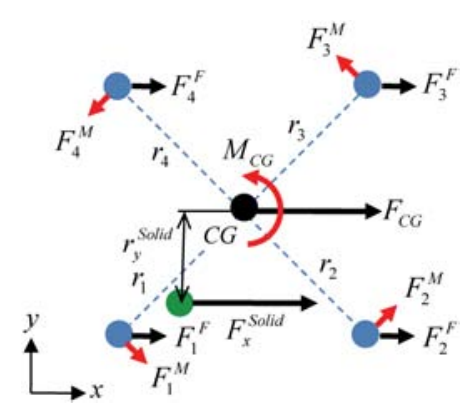

Figure 3. Concept of force distribution.

The force applied to the solid element node is transferred to the center of gravity $(C G)$ of the four nodes of the shell element. This transfer produces the equivalent force $F_{C G}$ and the equivalent moment $M_{C G}$ at the $C G$, that is

$$
\begin{aligned}
& F_{C G}=F_{x}^{\text {Solid }} \\
& M_{C G}=r_{y}^{\text {Solid }} F_{x}^{\text {Solid }}
\end{aligned}
$$

where $r_{y}^{\text {Solid }}$ denotes the $y$ component of the distance between the $C G$ and the solid element node $F_{C G}$ is divided and applied to each shell element node. The distributed force $F_{i}^{F}$ at the $i$ th node is given by

$$
F_{i}^{F}=\left(\frac{w_{i}}{\sum w_{k}}\right) F_{C G}=\left(\frac{w_{i}}{\sum w_{k}}\right) F_{x}^{\text {Solid }}
$$

where $w_{i}$ is a compensation factor and usually set to $1 . M_{C G}$ is divided and applied as a force to each shell element node. The distributed force $F_{i}^{M}$ at the $i$ th node is given by

$$
F_{i}^{M}=\left(\frac{w_{i} r_{i}}{\sum w_{k} r_{k}^{2}}\right) M_{C G}=r_{y}^{\text {Solid }}\left(\frac{w_{i} r_{i}}{\sum w_{k} r_{k}^{2}}\right) F_{x}^{\text {Solid }}
$$

where $r_{i}$ is the distance from the $C G$ to the $i$ th node. The total force distributed to each shell element node is obtained by computing the sum of the $F_{i}^{F}$ and $F_{i}^{M}$ vectors. Dividing the resultant force into the $x$ and $y$ components, we obtain

$$
\left\{\begin{array}{l}
F_{x}^{\text {Shell }} \\
F_{y}^{\text {Shell }}
\end{array}\right\}=\left\{\begin{array}{l}
\alpha_{x} \\
\alpha_{y}
\end{array}\right\} F_{x}^{\text {Solid }}
$$

where

$$
\begin{aligned}
& \left\{F_{x}^{\text {Shell }}\right\}=\left\{\begin{array}{llll}
F_{x 1}^{\text {Shell }} & F_{x 2}^{\text {Shell }} & F_{x 3}^{\text {Shell }} & F_{x 4}^{\text {Shell }}
\end{array}\right\}^{T} \\
& \left\{F_{y}^{\text {Shell }}\right\}=\left\{\begin{array}{llll}
F_{y 1}^{\text {Shell }} & F_{y 2}^{\text {Shell }} & F_{y 3}^{\text {Shell }} & F_{y 4}^{\text {Shell }}
\end{array}\right\}^{T} \\
& \left\{\alpha_{x}\right\}=\left\{\begin{array}{llll}
\alpha_{x 1} & \alpha_{x 2} & \alpha_{x 3} & \alpha_{x 4}
\end{array}\right\}^{T} \\
& \left\{\alpha_{y}\right\}=\left\{\begin{array}{llll}
\alpha_{y 1} & \alpha_{y 2} & \alpha_{y 3} & \alpha_{y 4}
\end{array}\right\}^{T}
\end{aligned}
$$

with

$$
\begin{aligned}
& \alpha_{x i}=\left(\frac{w_{i}}{\sum w_{k}}\right) \pm r_{y}^{\text {Solid }}\left(\frac{w_{i} r_{i}}{\sum w_{k} r_{k}^{2}}\right)_{x} \\
& \alpha_{y i}= \pm r_{y}^{\text {Solid }}\left(\frac{w_{i} r_{i}}{\sum w_{k} r_{k}^{2}}\right)_{y}
\end{aligned}
$$

The second term of $\alpha_{x i}$ is for the $x$ component of $F_{i}^{M}$ and $\alpha_{y}$ is for the $y$ component of $F_{i}^{M}$. The sign is determined by the relative position of the $i$ th node with respect to the $C G$.

The force distributed to each shell element node has a distinct quality as follows: In Figure 3, for the shell element nodes on the same side as the solid element node with respect to the $C G$ (lower side), a large force is distributed in the $x$ direction because $F_{i}^{F}$ and the $x$ component of $F_{i}^{M}$ are both positive. For the shell element nodes on the upper side, a small force is distributed because $F_{i}^{F}$ and the $x$ component of $F_{i}^{M}$ have opposite signs. This indicates that a large force in the $x$ direction is distributed to the shell element node close to the solid element node. For the distributed force in the $y$ direction, the magnitude of the force is approximately the same at the four shell element nodes, but the forces at the nodes on the left and right with respect to the $C G$, have opposite signs.

$\alpha_{x i}$ and $\alpha_{y i}$ in Equation (7) are used as the factors of the MPC equation. For other cases where a force is applied in the $y$ or $z$ direction to the solid element node, the factors can be determined in the same way.

\section{EQUATION OF MOTION}

For a whole spot welded structure, the equations of motion can be written as

$$
[M]\{\ddot{u}\}+[K]\{u\}=\{0\}
$$

where $[M]$ and $[K]$ are the mass and stiffness matrices and $\{u\}$ is the nodal displacement vector for the whole structure. Using Equation (1), we write the relationship between $\{u\}$ and $\left\{u^{A}\right\}$ as

$$
\{u\}=\left\{\begin{array}{c}
u^{A} \\
u^{O}
\end{array}\right\}=\left\{\begin{array}{c}
I \\
C_{O}
\end{array}\right\}\left\{u^{A}\right\}=[C]\left\{u^{A}\right\}
$$

where $\left\{u^{O}\right\}$ are the translations of the solid element nodes and $\left\{u^{A}\right\}$ are the translations and rotations excluding $\left\{u^{O}\right\}$. $[I]$ is the identity matrix and $\left[C_{O}\right]$ is the matrix that consists 
of the factors of the MPC equations. Substituting Equation (9) into Equation (8) and pre-multiplying the resulting equation by $[C]^{T}$, we get

$$
\begin{aligned}
& {\left[M^{A}\right]=[C]^{T}[M][C]} \\
& {\left[K^{A}\right]=[C]^{T}[K][C]}
\end{aligned}
$$

where $\left[M^{A}\right]$ and $\left[K^{A}\right]$ are the reduced mass and stiffness matrices. Equation (10) implies that the mass and stiffness of the solid element are distributed to the shell elements connected to the solid element (a group of shell elements is called a patch). The distributed stiffness is determined by the factors of the MPC equations which depend strongly on the configuration and the size of the patch, and the relative position between the patch and the solid element. Thus the modal properties of the spot welded structure are affected by the characteristics of the patch. The natural frequency $\omega$ and the mode shape $\{\phi\}$ are obtained from

$$
\left(\left[K^{A}\right]-\omega^{2}\left[M^{A}\right]\right)\{\phi\}=\{0\}
$$

\section{VERIFICATION OF THE ACM2 MODEL}

The characteristics of the ACM2 model are investigated using the simple welded structure shown in Figure 4. The structure consists of two steel plates with the same dimensions (231 $\mathrm{mm}$ long $\times 204 \mathrm{~mm}$ wide $\times 1.6 \mathrm{~mm}$ thick) joined by three spot welds. The overlap length of the two plates is $30 \mathrm{~mm}$. Three spot welds are lined up on the center of the overlap in the $x$ direction, one of which is centrally located in the $y$ direction while the others are located $21 \mathrm{~mm}$ away from the edge of the plate.

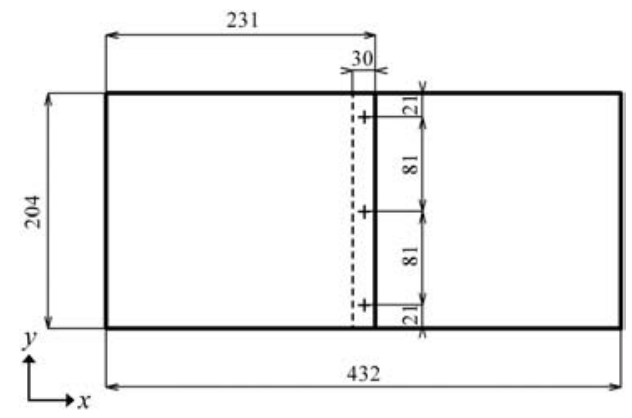

Figure 4. Spot welded structure.

Before investigating the characteristics of the ACM2 model, we verified the validity of the finite element model developed in this paper. For this purpose, a test structure with the same dimensions as in Figure 4 was constructed and the frequency response functions (FRFs) were measured. The FRFs were then compared with those predicted by the FE analysis.
Figure 5 shows the test structure suspended using rubber bands to simulate the free-free boundary condition. The accelerometers are placed at the two corners of the plate. The structure was impacted at the upper right corner using an impact hammer and the excitation force and the response accelerations were measured. The FRFs were then calculated. For the test structure, the spot weld diameter of the three spot welds was approximately $7 \mathrm{~mm}$.

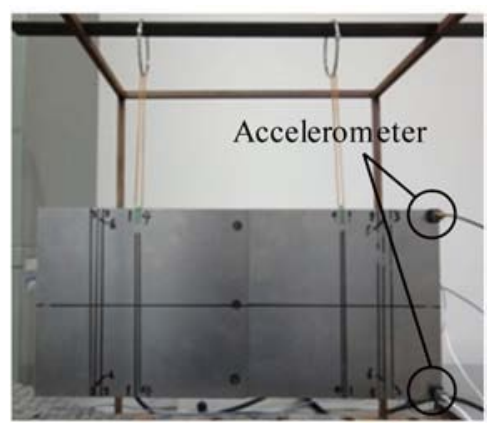

Figure 5. Experimental setup and accelerometer locations.

\section{FINITE ELEMENT ANALYSIS}

The ACM2 model was developed using NASTRAN. In this paper, we create a finite element (FE) model for spot welds that has the same features as the ACM2 model using ANSYS. For a FE model of the spot welded structure shown in Figure $\underline{4}$, the upper and lower plates were modeled with shell elements (Shell 181). The spot welds were modeled in two different ways: The first modeled the spot weld using a single cylindrical beam element (Beam 188, $7 \mathrm{~mm}$ diameter and 1.6 $\mathrm{mm}$ high), called the beam model. The second modeled the spot weld using a single solid element (Solid 185, $6 \mathrm{~mm}$ square and $1.6 \mathrm{~mm}$ high), which is the ACM2 model. For the upper and lower plates, the mesh size was $3 \mathrm{~mm}$ square or half of the solid element size $6 \mathrm{~mm}$ square. For the plates and the spot welds, the material properties were Young's modulus $E=217 \mathrm{GPa}$, mass density $\rho=7840 \mathrm{~kg} / \mathrm{m}^{3}$ and Poisson's ratio $v=0.28$. The mass of the accelerometer $(5.3 \mathrm{~g})$ was taken into consideration as a lumped mass. The natural frequencies and mode shapes were calculated under the freefree boundary condition identical to that in the experiment.

Figure 6 shows the first 12 flexible mode shapes, excluding the six rigid body mode shapes, when the ACM2 model is used as the spot weld model. It can be seen that the mode shapes for modes 2, 3, 6, 8 and 11 have a nodal line along the three spot welds. This indicates that these mode shapes produce a small strain in the area of the spot welds. 

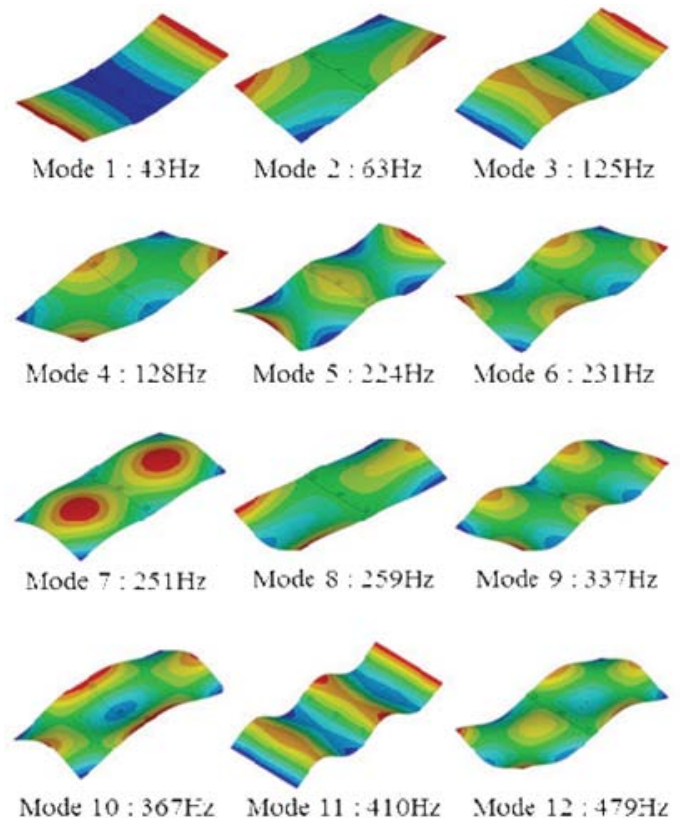

Figure 6. First twelve flexible mode shapes.

\section{COMPARISON OF THE MEASURED AND FE PREDICTION RESULTS}

Figure 7 shows the comparison of the driving point FRFs measured and predicted by the FE analysis. The resonance peaks for the beam model (dotted line) are lower than those for the measured FRF (solid line), especially, for modes 9,10 and 11. This indicates that the beam model produces imprecise results and underestimates the stiffness of a real spot welded joint. In contrast, the resonance peaks for the ACM model (broken line) are in good agreement with those for the measured FRF. Since the ACM2 model shows good performance, we investigate the dynamic characteristics of this model in detail.

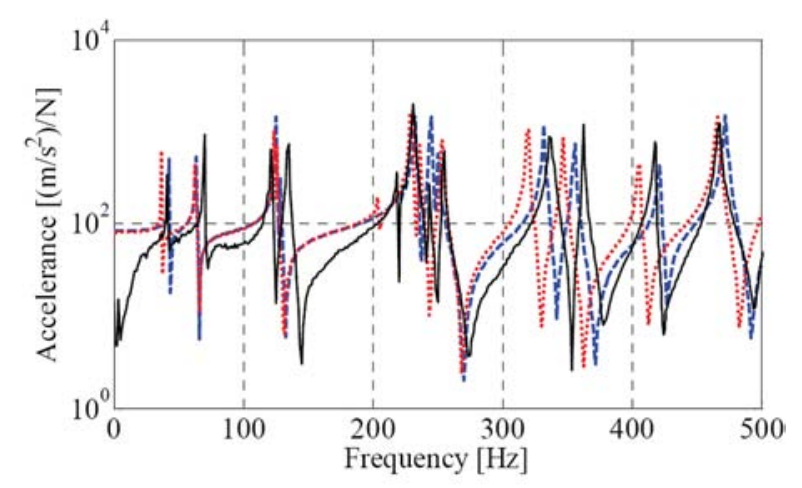

Solid line: Measured, Broken line: ACM2 model, Dotted line: Beam model

Figure 7. Comparison of the measured and FEA predicted frequency response functions.

\section{CHARACTERISTICS OF THE ACM2 MODEL}

For the ACM2 model, the configuration and size of a patch and the relative position of the patch to the solid element depend strongly on the mesh pattern surrounding the solid element. Since this changes the vibration properties of the spot welded structure, we investigate the effect on the modal properties of the mesh pattern in the area of the spot weld.

\section{AUTOMATICALLY GENERATED PATCH}

Automotive manufacturers usually generate the ACM2 model using the automated spot weld modeling program. This program detects the shell element which has a solid element node lying inside and connects the solid element node to the four shell element nodes with four RBE3 elements. In this section, the characteristics of the ACM2 model with an automatically generated patch are examined.

\section{Effect of Patch Size}

The effect of the patch size on the natural frequency is examined. Assume that the patch consists of four shell elements of the same size and square shape, the centers of the patch and the solid element are coincident and the mesh pattern of the upper and lower plates is the same (Figure 8(a) and is called the coincidence patch). The rest of the plate is meshed with the same mesh size as in the patch but nonsquare elements occur along each edge of the plate.

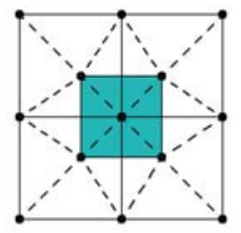

(a) Coincidence patch

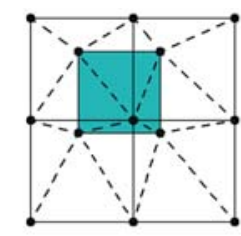

(b) Non-coincidence patch
Figure 8. Top view of the ACM2 model.

As the element size was changed from 3 to $15 \mathrm{~mm}$, the natural frequencies were calculated. Figure 9 shows the change in the natural frequency with the change in the element size. The vertical axis represents the percentage change relative to the reference natural frequency when the patch consists of four $3 \mathrm{~mm}$ square elements and the shell element nodes are directly connected to the solid element nodes. It is seen that the natural frequencies for all modes increase slowly with the element size. This indicates that the patch size significantly affects the change in the natural frequency of the spot welded structure. 


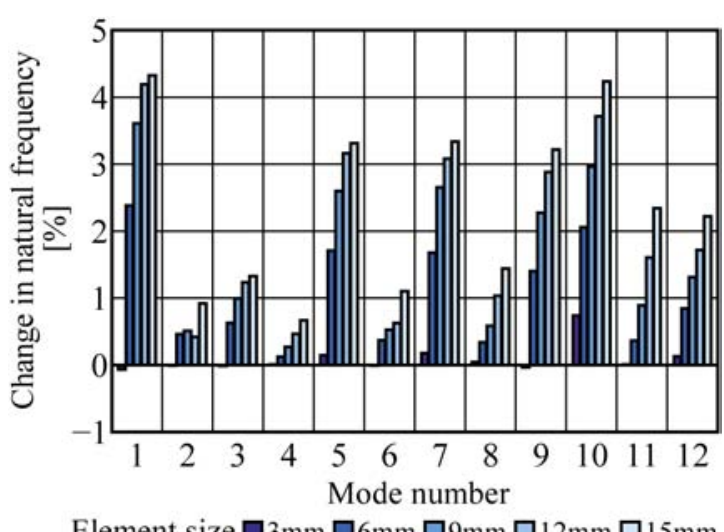

Element size $\square 3 \mathrm{~mm} \square 6 \mathrm{~mm} \square 9 \mathrm{~mm} \square 12 \mathrm{~mm} \square 15 \mathrm{~mm}$

Figure 9. Percentage change in the natural frequency: coincidence of the centers of patch and solid element.

For the vibration analysis of a large scale model such as an automotive body, a relatively course mesh can be used because reducing the element size is difficult due to the computational effort required. One way of obtaining an improved agreement between the FE model prediction and the experiment is to reduce Young's modulus in the patch intentionally. In the case of a monotonic increase in the natural frequencies as shown in Figure 9, the modification is valid because the reduction ratio of Young's modulus is easily estimated.

\section{Effect of the Relative Position of the Patch and the}

\section{$\underline{\text { Solid Element }}$}

In building the FE model of a whole structure, the coincidence patch as shown in Figure 8(a) is rarely generated. In this section, we deal with the case where the centers of the patch and the solid element are not coincident (Figure 8(b) and is called the non-coincidence patch) and examine its effect on the natural frequency. For the spot welded structure in Figure 4, the upper and lower plates were meshed from the right edge in the $x$ direction and from the bottom edge in the $y$ direction. As a result, the non-coincidence patches were generated and non-square shaped elements occurred on the right edge in the $x$ direction and on the top edge in the $y$ direction.

The mesh size of each plate was chosen as 3, 6, 9, 12 and 15 $\mathrm{mm}$, that is, the element size in the patch is the same as in the previous section. Figure 10 shows the change in the natural frequency with the change in the element size. The vertical axis represents the percentage change relative to the reference natural frequency in the previous section. Comparing the results in Figure 9, we can see that the increases in the natural frequencies are small when the mesh size increases from 3 to $9 \mathrm{~mm}$ and then the natural frequencies rapidly increase at the $12 \mathrm{~mm}$ element size. In addition, the changes are different from mode to mode at the $15 \mathrm{~mm}$ element size; in particular, the natural frequency for mode 1 decreases from the natural frequency of the $12 \mathrm{~mm}$ element size.

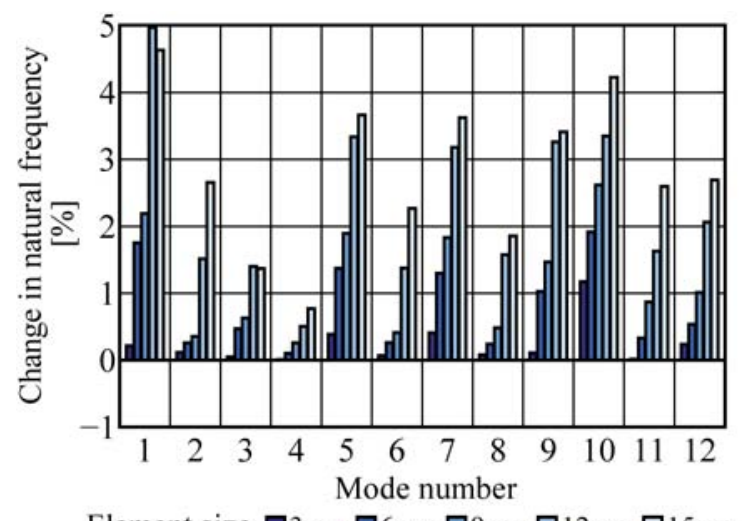

\section{Figure 10. Percentage change in the natural frequency: non-coincidence of the centers of patch and solid element.}

For the non-coincidence patch, in addition to the patch configuration as shown in Figure 8(b), a rectangle-shaped patch as shown in Figure 11 occurs when the element size is $9 \mathrm{~mm}$ or more. This is because the patch consists of less than four shell elements. As a result, the characteristics of the ACM2 model vary widely and the natural frequencies do not change monotonically unlike those for the coincidence patch. Thus the variation in the natural frequency is large and it is difficult to adjust the difference between the FE model and the real structure by intentionally reducing Young's modulus in the patch.

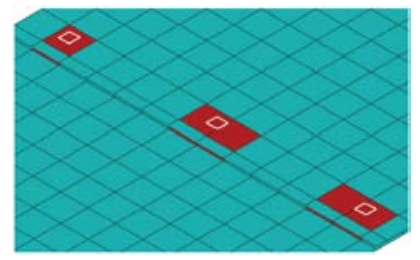

Figure 11. Patch configuration: $15 \mathrm{~mm}$ element size.

\section{EFFECT OF PATCH SIZE ONLY}

Automotive manufacturers usually generate the ACM2 model using the automated spot weld modeling program. This program detects the shell element in which a solid element node is lying and connects the solid element node to the shell element nodes. In this case, the patch size depends on the shell element size and, as a result, the influence of the enlargement of shell elements is included. To examine the effect of the patch size only, we kept the element size $3 \mathrm{~mm}$ square and changed the patch size by building the patch manually. Figure 12 shows the coincidence patch that consists of four or more shell elements. 


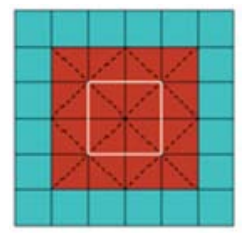

Figure 12. Top view of the ACM2 mode: square-shaped patch.

As the patch size was changed from 6 to $24 \mathrm{~mm}$, the natural frequencies were calculated. Figure 13 shows the change in the natural frequency with the change in the patch size. The vertical axis represents the percentage change relative to the reference natural frequency in the previous section. It is seen that the natural frequencies increase with the patch size which is the same tendency as in Figure 9. However, the absolute changes in the natural frequencies are smaller than those in Figure 9 because the influence of the enlargement of the shell element is eliminated. The changes in the natural frequencies for mode 2, 4, 6, 8 and 11 are very small and this indicates that these modes are little affected by the patch size. In contrast, the natural frequencies for modes 1, 5, 7, 9 and 10 increase rapidly with the patch size and this indicates that these modes are greatly affected by the patch size.

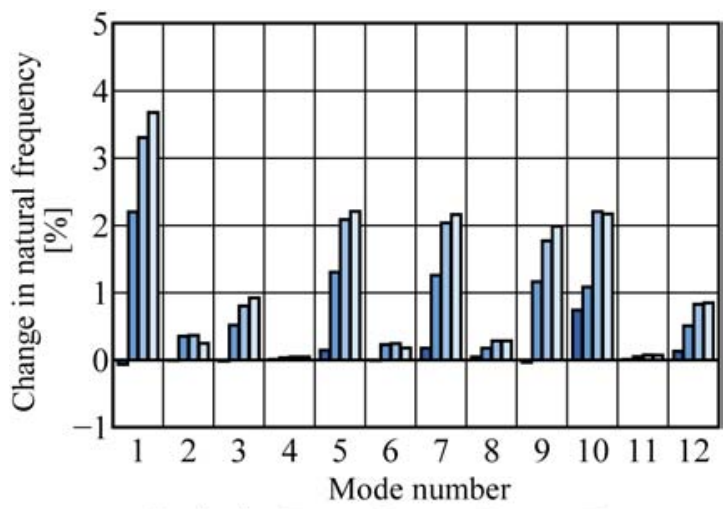

Patch size $\square 6 \mathrm{~mm} \square 12 \mathrm{~mm} \square 18 \mathrm{~mm} \square 24 \mathrm{~mm}$

Figure 13. Percentage change in the natural frequency: square-shaped patch.

As shown in Equation (10), the stiffness of the solid element is distributed between the shell element nodes and the distribution of the stiffness is based on the distribution of the force. For the coincidence patch, the force distributed to the patch center becomes noticeably larger with the patch size. This is because the distance between the patch center and the solid element node becomes shorter for all four nodes. As a result, the stiffness at the patch center increases with the patch size.

\section{CHANGE IN THE STIFFNESS AT THE PATCH CENTER}

In the previous section, we mentioned that the change in the natural frequency is due to the change in the stiffness at the patch center. To verify this, the relationship between the stiffness at the patch center and the patch size is examined. Figure 14 shows an analytical model used in this section. This model has a single spot weld with a square-shaped patch. The upper and lower plates were meshed with $3 \mathrm{~mm}$ square shell elements. When the tensile force in the $z$ direction was applied to the patch center, the displacement in the $z$ direction was measured at the force point and the equivalent stiffness was calculated as the ratio of the force value and the displacement.

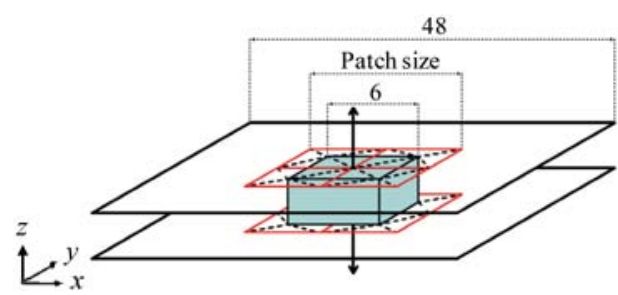

Figure 14. Model for the tensile test.

Figure 15 shows the change in the equivalent stiffness as the patch size is changed from 6 to $24 \mathrm{~mm}$. The scale in the vertical axis is normalized by the equivalent stiffness for the $6 \mathrm{~mm}$ patch size. The equivalent stiffness increases with the patch size which is the reason for the increase in the natural frequency.

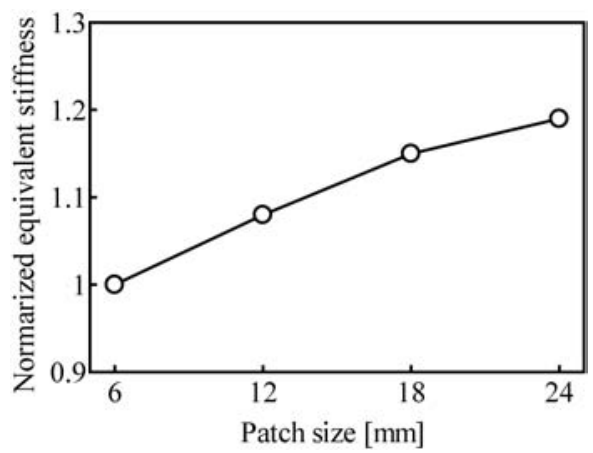

Figure 15. Relationship between the equivalent stiffness and the patch size.

\section{APPROPRIATE ELEMENT SIZE}

In the previous section, we demonstrated that the patch configuration varies widely depending on the meshing when the element size is large. As a result, the variation in natural frequency is large. In this section, we find an appropriate element size that produces a small variation in natural frequency. 
The automated spot weld modeling program is used here. As mentioned in the previous section, the patch often consists of less than four shell elements and the rectangle-shaped patch occurs for the element size of $9 \mathrm{~mm}$ or more. Thus we examined the variation for the element sizes of 3 and $6 \mathrm{~mm}$. The patch configuration was changed by moving the starting location for meshing while the element size was fixed. Nonsquare elements occurred along some edges of the plate. Figure 16 shows the patch configurations considered. The patch configuration of the three spot welds for the $3 \mathrm{~mm}$ element size is the same while the patch configurations for the $6 \mathrm{~mm}$ element size are different.

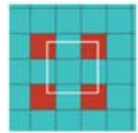

Case 1

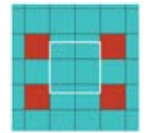

Case 2 (a). $3 \mathrm{~mm}$ element size

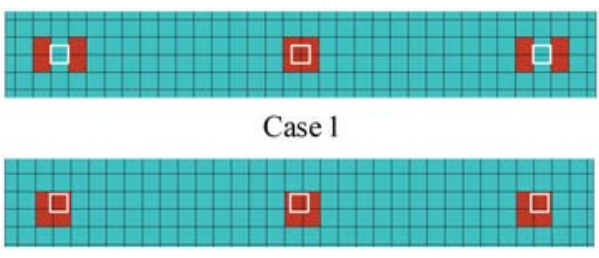

Case 2

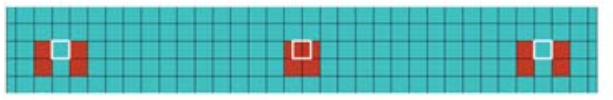

Case 3

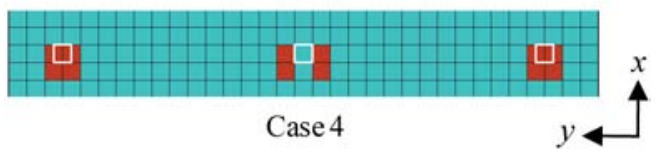

(b). $6 \mathrm{~mm}$ element size
Figure 17 shows the natural frequency for each patch configuration. The vertical axis represents the percentage change relative to the reference natural frequency when the patch configuration is the coincidence patch in Figure 8(a). For the $3 \mathrm{~mm}$ element, the natural frequencies are higher than the references for all cases except case 4 . This is because the distance between the center of the patch and the solid element is small. In addition the four shell elements are widely arranged and this overestimates the true size. However, the wider arrangement such as case 4 results in small changes in the natural frequencies. For the $6 \mathrm{~mm}$ element, unlike the 3 $\mathrm{mm}$ element, the natural frequencies are lower than the references for all cases except case 1. This is because the elements are widely arranged in the $y$ direction and the distance between the center of the patch and the solid element is large. These cause the stiffness at the patch center to decrease. Although the difference between cases 1 and 3 is the distance between the center of the patch and the solid element, this produces a large decrease in the natural frequency. Thus the distance between the center of the patch and the solid element considerably affects the change in the natural frequency.

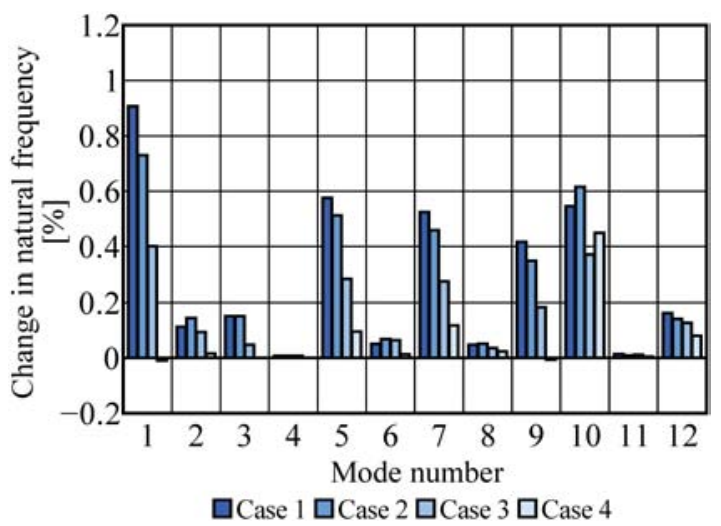

(a). $3 \mathrm{~mm}$ element size

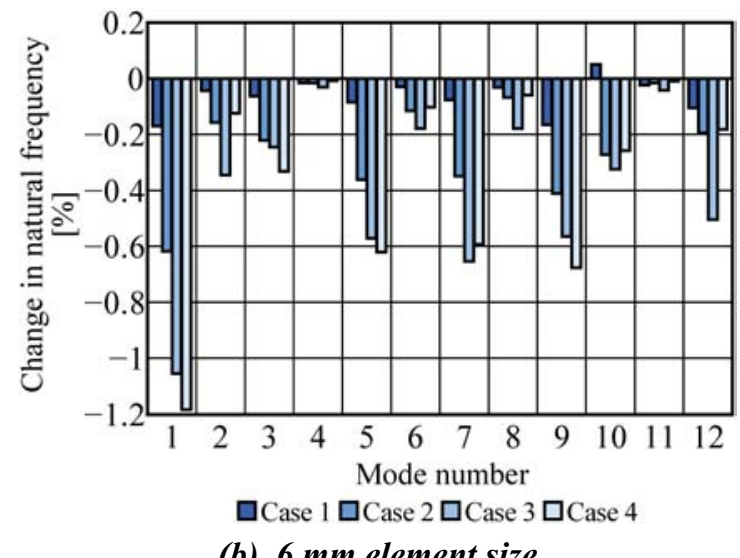

(b). $6 \mathrm{~mm}$ element size

Figure 17. Percentage change in the natural frequency.

The difference from the reference is small for both 3 and 6 $\mathrm{mm}$ elements and the maximum difference is approximately 1 $\%$ for mode 1 . Thus when the patch is composed of shell elements which are the same size as or smaller than the solid element which is determined by the spot weld diameter, the patch consists of four shell elements. As a result, the variation in the natural frequency is small.

\section{CONCLUSIONS}

In this paper, we focused on the ACM2 (Area Contact Model 2) model as a simplified spot weld model for vibration analysis using FEA and explained its theoretical background. In addition, the dynamic characteristics, especially the effect of the mesh pattern surrounding a spot weld on the modal properties (natural frequency and mode shape) of a spot welded structure, was examined in detail. As an example, a structure that consisted of two steel plates joined by three spot welds was used. The main conclusions of this paper are as follows. 
For the MPC (multi-point constraint) equation using the RBE3 (Rigid Beam Element 3) element, the factors of the MPC equation are large at the shell element node close to the solid element node. According to the factors, the stiffness of the solid element is distributed between the shell element nodes.

When the patch (group of shell elements in the ACM2 model) consists of four shell elements and the centers of the patch and the solid element are coincident, the stiffness at the patch center increases with the patch size and as a result, the natural frequencies increase.

When the shell element size in the patch is large, a patch often consists of less than four shell elements and a rectangleshaped patch occurs. This causes the natural frequencies to vary widely.

The large difference between the center of the patch and the solid element decreases the natural frequencies.

When the patch is composed of shell elements, which are the same size as or smaller than the solid element that is determined by the spot weld diameter, the variation in the natural frequencies is small.

\section{REFERENCES}

1. Radaj, D., Zheng, Z. and Mohrmsnn, W., "Local stress parameters at the weld spot of various specimens," Engineering Fracture Mechanics, 37(5): 933-951, 1990.

2. Chen, W. and Deng, X., "Performance of shell elements in modeling spot welded joints," Finites Elements in Analysis and Design, 35: 41-57, 2000.

3. Heiserer, D., Charging, M. and Sielaff, J., "High performance, process oriented, weld spot approach," Proceedings of the 1st MSC Worldwide Automotive User Conference, 1999.

4. Jonscher, A., Lewerenz, M. and Luhrs, G., "MSC.Nastran's new spot weld element in the CAE-process," Proceedings of the 2nd MSC Worldwide Automotive User Conference, 2000.
5. Lardeur, P., Lacouture, E. and Blain, E., "Spot weld modeling techniques and performances of finite element models for the vibration behavior of automotive structures," Proceedings of ISMA25, 2000.

6. Palmonella, M., Friswell, M.I., Mares, C. and Mottershead, J.E., "Improving spot weld models in structural dynamics," Proceedings of the ASME 2003 Design Engineering Technical Conferences and Computers and Info in Engineering Conference, 1-10, 2003.

7. Palmonella, M., Friswell, M.I., Mottershead, J.E. and Lees, A.W., "Finite element models of spot welds in structural dynamics: review and updating," Computer and Structures, 83: 648-661, 2005.

8. UGS Corp., NX Nastran Element Library Reference Manual, 211-213, 2005.

\section{CONTACT INFORMATION}

Fumiyasu KURATANI

Professor

Department of Mechanical Engineering, University of Fukui

3-9-1 Bunkyo, Fukui 910-8507, Japan

Tel/Fax: +81-776-27-8538

kuratani@mech.u-fukui.ac.jp

Kazuhei MATSUBARA and Takashi YAMAUCHI

Graduate School of Engineering, University of Fukui

\section{ACKNOWLEDGMENTS}

This work was supported by the Grant-in-Aid for Scientific Research (C) (21560237).
The Engineering Meetings Board has approved this paper for publication. It has successfully completed SAE's peer review process under the supervision of the session organizer. This process requires a minimum of three (3) reviews by industry experts.

All rights reserved. No part of this publication may be reproduced, stored in a retrieval system, or transmitted, in any form or by any means, electronic, mechanical, photocopying, recording, or otherwise, without the prior written permission of SAE.

ISSN 0148-7191
Positions and opinions advanced in this paper are those of the author(s) and not necessarily those of SAE. The author is solely responsible for the content of the paper.

SAE Customer Service:

Tel: 877-606-7323 (inside USA and Canada)

Tel: 724-776-4970 (outside USA)

Fax: 724-776-0790

Email: CustomerService@sae.org

SAE Web Address: http://www.sae.org

Printed in USA 